ARTICLE

https://doi.org/10.1038/s41467-020-14364-3

\title{
Vacuum Rabi splitting of a dark plasmonic cavity mode revealed by fast electrons
}

Ora Bitton ${ }^{1,5}$, Satyendra Nath Gupta ${ }^{2,5}$, Lothar Houben ${ }^{1,5}$, Michal Kvapil (1) ${ }^{3,4,5}$, Vlastimil Krápek (iD ${ }^{3}$, Tomáš Šikola ${ }^{3,4}$ \& Gilad Haran (1) ${ }^{2 *}$

Recent years have seen a growing interest in strong coupling between plasmons and excitons, as a way to generate new quantum optical testbeds and influence chemical dynamics and reactivity. Strong coupling to bright plasmonic modes has been achieved even with single quantum emitters. Dark plasmonic modes fare better in some applications due to longer lifetimes, but are difficult to probe as they are subradiant. Here, we apply electron energy loss (EEL) spectroscopy to demonstrate that a dark mode of an individual plasmonic bowtie can interact with a small number of quantum emitters, as evidenced by Rabi-split spectra. Coupling strengths of up to $85 \mathrm{meV}$ place the bowtie-emitter devices at the onset of the strong coupling regime. Remarkably, the coupling occurs at the periphery of the bowtie gaps, even while the electron beam probes their center. Our findings pave the way for using EEL spectroscopy to study exciton-plasmon interactions involving non-emissive photonic modes.

\footnotetext{
${ }^{1}$ Chemical Research Support Department, Weizmann Institute of Science, POB 26, 7610001 Rehovot, Israel. ${ }^{2}$ Department of Chemical and Biological Physics, Weizmann Institute of Science, POB 26, 7610001 Rehovot, Israel. ${ }^{3}$ Central European Institute of Technology, Brno University of Technology, Purkyňova 123, 61200 Brno, Czech Republic. ${ }^{4}$ Institute of Physical Engineering, Brno University of Technology, Technická 2, 616 69 Brno, Czech Republic.

${ }^{5}$ These authors contributed equally: Ora Bitton, Satyendra Nath Gupta, Lothar Houben, Michal Kvapil. *email: gilad.haran@weizmann.ac.il
} 
C avity quantum electrodynamics deals with the interaction of quantum emitters with the electromagnetic (EM) fields within optical cavities ${ }^{1}$. When this interaction is large enough to reach the so-called strong coupling limit, coherent excitations comprising both cavity modes and emitter modes are generated by excitation with resonant EM radiation. These coherent excitations are manifested by phenomena such as vacuum Rabi splitting, namely the appearance of two branches in optical spectra. Strong coupling is of importance for many quantum technological applications, such as quantum information processing 2,3 and quantum communication ${ }^{4,5}$. In recent years, it has been shown that strong coupling to EM modes can also be used to modify photophysics and chemical reaction dynamics ${ }^{6,7}$.

Plasmonic cavities (PCs) are nanometric structures that focus EM fields to sub-diffraction volumes through the effect of surface plasmon excitations ${ }^{8,9}$. The deep sub-diffraction volumes of the EM modes of PCs make them attractive for quantum optical applications. PCs are often made of metallic nanostructures, and rely on the concentration of the EM energy in gaps between particles or at sharp tips or corners. Due to the lossy nature of metals, PCs typically have only moderate quality factors, but their ultra-small mode volumes facilitate strong coupling even under ambient conditions ${ }^{10}$. Indeed, we recently showed that individual PCs can couple to individual or just a few quantum emitters ${ }^{11}$. In particular, we coupled semiconductor quantum dots (QDs) to plasmonic bowties, and observed vacuum Rabi splitting in light scattering spectra. Similar observations were reported more recently by other labs using both scattering and emission spectra measured from individual devices ${ }^{12,13}$.

PCs can sustain a set of multipolar resonances, some of which are bright while others are dark and subradiant ${ }^{8}$. It is typically the lowest energy, bright dipolar resonance that is used for coupling to quantum emitters. However, dark modes might also be of significant interest for quantum optical studies, particularly since they are expected to have longer lifetimes than bright modes and therefore should be able to store EM energy for longer times ${ }^{14,15}$. It is difficult, though, to excite and probe dark modes of individual plasmonic structures, due to their inability to couple radiatively to the far-field.

Electron energy loss (EEL) spectroscopy is an electron microscopy method that can access the EM near-field and yield the complete spectrum of plasmonic modes in nanostructures with a very high spatial resolution ${ }^{16}$. EEL spectroscopy has been applied extensively to study both bright and dark resonances of various plasmonic structures ${ }^{17-20}$. It probes the out-of-plane component of the electric field around a plasmonic device ${ }^{16}$, and the EEL signal is closely related to the optical extinction spectrum of a plasmonic structure ${ }^{16,21,22}$.

Here, we show that EEL spectroscopy in a scanning transmission electron microscope (STEM) can be used to probe the coupling of silver bowtie PCs with QDs residing in their vicinity. We demonstrate vacuum Rabi splitting in EEL spectra due to coupling between the QDs and the dark mode of the PCs. In contrast to expectation, the strongest coupling occurs at the periphery of the PC gaps. EM simulations allow us to attribute this finding to the non-uniform distribution of the EM field of the dark mode.

\section{Results}

Fabrication of plasmonic bowties coupled to QDs. We fabricated silver bowties using electron beam lithography on $\mathrm{SiO}_{2}$ membranes, $18 \mathrm{~nm}$ thick, and inserted QDs into their gaps and around them. For more details, see Methods section.
Figure 1a shows an elemental map of such a device, obtained by energy dispersive $\mathrm{x}$-ray spectroscopy (EDS). The bowtie is protected by a thin alumina layer against oxidation and the CdSe/ $\mathrm{ZnS}$ QDs are seen around it.

Bright and dark plasmonic modes of a bare bowtie revealed by EEL spectra. The EEL spectrum measured from a PC depends on the position of the electron beam, as different positions excite a different combination of plasmonic modes ${ }^{18}$. Figure $1 \mathrm{~b}$ shows EEL spectra measured at two different positions on a bare PC devoid of QDs. When the electron beam interacts with the outer edge of the bowtie (orange dot in the inset of Fig. 1b), the lowestenergy excited plasmonic mode is found at $1.8 \mathrm{eV}$ (Fig. 1b, orange spectrum). From the calculated charge density map obtained at this frequency (Fig. 1c), it is clear that this is the lowest-energy bright longitudinal dipolar mode of the PC. In contrast, when the electron beam passes through the center of the PC gap (red dot in the inset of Fig. 1b), a mode at $2.1 \mathrm{eV}$ is excited, whose charge distribution identifies it as the lowest-energy dark mode. This mode is formed by an antiparallel combination of the dipolar plasmons of the individual prisms of the bowtie, carries a zero net dipole moment (Fig. 1c) ${ }^{18}$ and is subradiant ${ }^{23}$. Additional modes excited with the electron beam at other positions are shown in Supplementary Figs. 1 and 2, and will not be further discussed below.

Figure 1d, e shows EEL maps at certain energy slices, each corresponding to one of the peaks shown in Fig. 1b. Thus, for example, the map at energies between 1.76 and $1.85 \mathrm{eV}$ corresponds to the bright dipolar mode discussed above. Interestingly, the EEL signal, which (as noted above) is sensitive to the out-of-plane component of the EM field, is stronger on the outer edge than in the gap. On the other hand, for the energy slice around $2.1 \mathrm{eV}$ (corresponding to the dark dipolar mode), the outof-plane field is much more confined in the gap region than at the outer edges.

EELS spectra of bright and dark plasmonic modes coupled to QDs. When we introduce into PC gaps QDs whose lowest optical transition (the so-called band-edge exciton ${ }^{24}$ ) is in resonance with the bright dipolar mode, EEL spectra show a clear evidence of a Rabi splitting. Two such spectra are presented in Fig. $2 b$, d, and ADF-STEM images of the devices are shown in Fig. 2a, c. The Rabi splitting values in these devices are 200 and $150 \mathrm{meV}$, respectively. The splitting is an indication of the formation of plasmon-exciton polaritons, new states that mix (to a different degree, depending on the coupling strength) the exciton and plasmon states. To obtain the coupling strength characterizing each spectrum, we fitted the spectra to the coupled oscillator model $^{25}$. Since, as already noted, it has been shown that the EELS signal is closely related to the optical extinction spectrum $^{16,21}$, we used the expression for extinction derived from the coupled oscillator model (see Methods) ${ }^{25}$. We find coupling strengths of $105 \pm 2 \mathrm{meV}$ and $68 \pm 3 \mathrm{meV}$. Similar coupling strengths were reported in our previous work and in others' for a small number of QDs ${ }^{11-13}$. An EM simulation of the EELS signal using the boundary-element method (BEM) ${ }^{26,27}$ with four QDs positioned in the PC gap yields a similar splitting $(120 \mathrm{meV})$ in the lowest energy mode (Fig. 2e, f).

Remarkably, when QDs with a band-edge exciton at $2 \mathrm{eV}$ are introduced into PC gaps, EEL spectra measured with the beam within the gaps are also found to split (Fig. 3), demonstrating that the QDs can also couple to the dark dipolar mode. The three panels of Fig. 3 demonstrate coupling to the dark mode, with Rabi splitting values of 160,150 and $100 \mathrm{meV}$. Fits to the coupled 


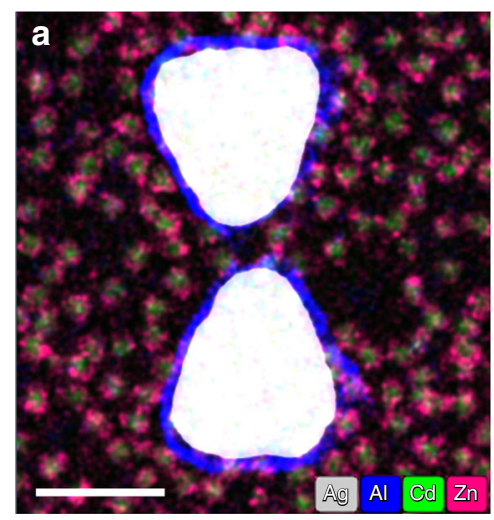

C

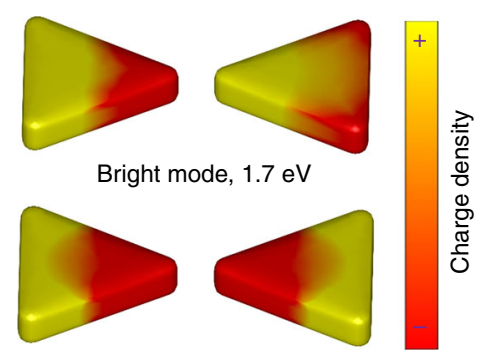

Dark mode, $2.04 \mathrm{eV}$

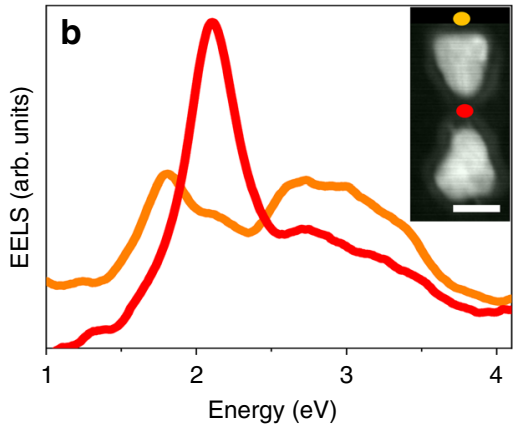

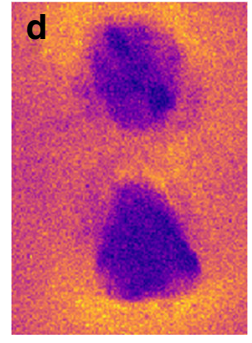

$1.76-1.85 \mathrm{eV}$

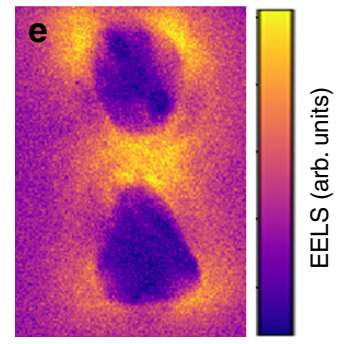

$2.05-2.15 \mathrm{eV}$

Fig. 1 Plasmonic bowties coupled with quantum dots. a Electron micrograph of a plasmonic bowtie with QDs in its vicinity. The image is a color-coded superposition of elemental maps taken by EDS in the STEM. The color key is shown at the bottom of the image. The scale bar represents 50 nm. $\mathbf{b}$ EEL spectra of a bare bowtie measured at the two locations indicated by matching colored dots in the inset, which is an annular dark-field (ADF) STEM image of the device. The scale bar represents $50 \mathrm{~nm}$. c Simulated charge-density maps for the bright and dark dipolar modes at the indicated energies. d, e Experimental EEL maps for the bright (d) and dark (e) modes, constructed at the indicated energy ranges.

oscillator model give values of $83 \pm 2,75 \pm 2$ and $57 \pm 1 \mathrm{meV}$, respectively, for the coupling strength.

Coupling takes place at the periphery of the bowtie gap. In order to understand the mechanism of the exciton-plasmon interaction involving the dark mode, we performed BEM simulations of the EEL spectra of coupled devices. Unexpectedly, with four and even eight QDs positioned in the PC gap as above, no splitting was observed in the simulated spectrum (Fig. 4a). However, when ten additional QDs were added at the periphery of the PC gap rather than its center (Fig. 4b), a clear splitting was observed in the spectrum, implying that the QDs at the periphery contribute more to the exciton-dark mode interaction than the ones at the center. Indeed, a simulation with QDs positioned only at the gap periphery (Fig. 4c) showed a similar splitting. To shed more light on this surprising observation, we plot a map of the simulated total electric field distribution around a bare PC at $2 \mathrm{eV}$, shown in Fig. $4 \mathrm{~d}$ as a projection on the $x-y$ plane. It is clearly seen that the electric field at the center of the gap, and even near the tips of the two prisms forming the bowtie, is very small. On the other hand, a very high field is observed at the periphery of the gap, comparable to the highest field in the bright mode map (Supplementary Fig. 2a). Since the coupling strength is linearly proportional to the electric field, it is expected that QDs that reside at the center of the gap will not couple at all with the dark dipolar mode, while QDs at the periphery will strongly couple with it. Notably, the out-of-plane component of the electric field has a small contribution in the gap (Fig. 4e, showing a projection on the $x-y$ plane), $\sim 50$ smaller than the total field, but this small contribution is enough to couple to the electron beam and generate an EEL signal.
To verify that indeed coupling at the periphery of the gap is responsible for the observed Rabi splitting, we looked for devices in which the QDs were not located at the center of the gap. Figure 3a shows an example of such a situation; even while the electron beam probes the center of the device, there are no QDs there, and the QDs situated at the periphery couple to the PC, leading to the appearance of Rabi splitting in the EEL spectrum. This observation directly confirms the simulation results. An additional such example is shown in Supplementary Fig. 3.

\section{Discussion}

We reported here vacuum Rabi splitting in EEL spectra that can be traced to coupling between quantum emitters and a dark plasmonic mode of an individual PC. This interaction cannot be accessed optically in the far field, as the dark mode is not radiant. However, the ability to excite the dark mode with an electron beam opens the way to observe the respective PC-QD interaction in EEL spectra. EEL spectroscopy has been used recently to probe strong coupling to bright plasmonic modes of individual plasmonic structures ${ }^{28,29}$. While the merit of dark modes for strong coupling phenomena has been discussed theoretically 30,31 , we are not aware of any previous experimental study of the coupling to a dark mode at the level of a single PC. (One recent study exploited the coupling of a dark lattice resonance mode of a plasmonic array to a layer of molecules for lasing ${ }^{32}$.) The coupling effect depends on the local distribution of the EM field in the PC gap region. Counterintuitively, it is not the gap center that provides the largest coupling, even though this is exactly where the combined excitations are probed.

It is instructive to ask whether the coupling strength values we observe here put our PC-QD systems in the strong coupling regime. To that end, we compare our measured $g$ values to two 

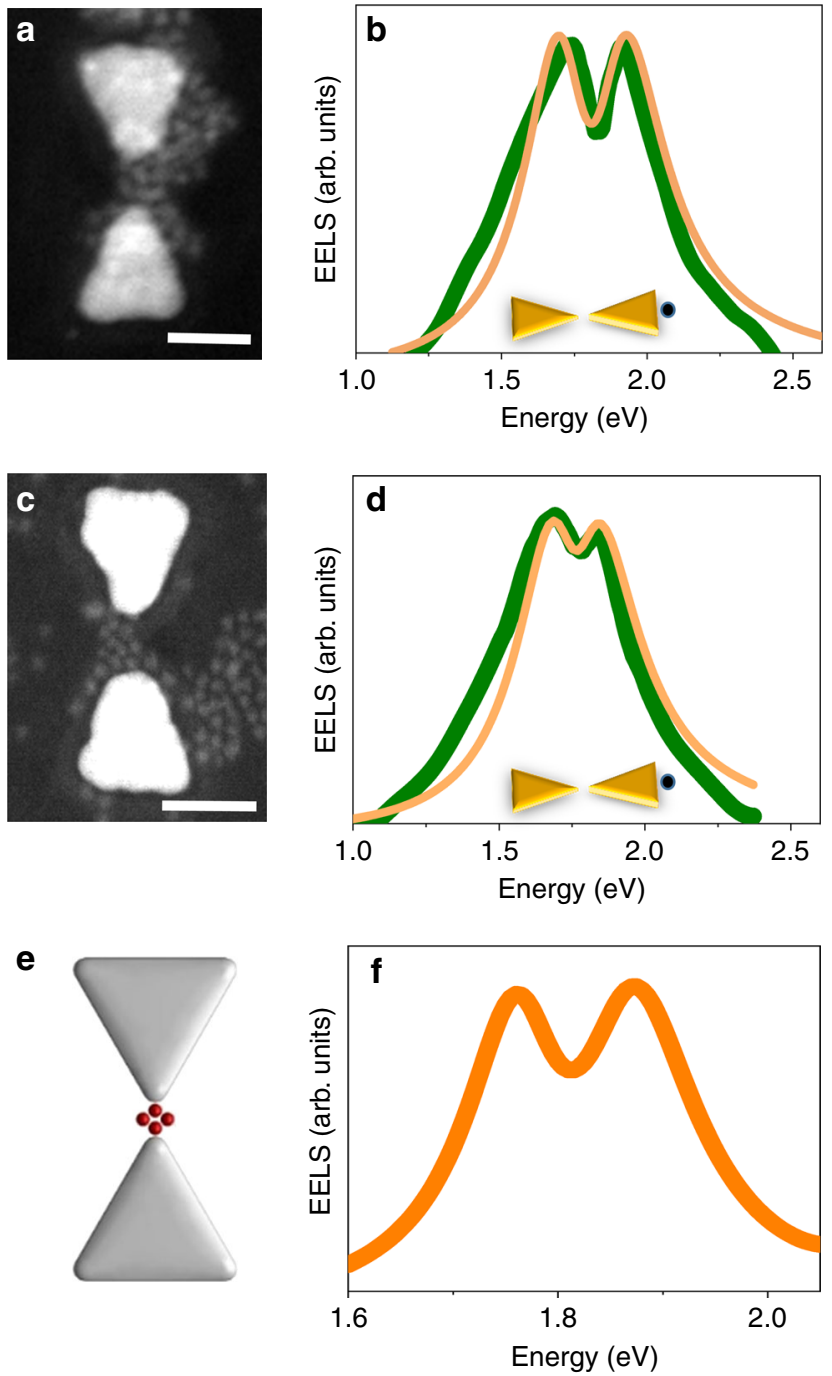

Fig. 2 Coupling with the bright dipolar mode. a, c ADF-STEM images of two devices loaded with QDs with an exciton energy of $1.8 \mathrm{eV}$. The scale bars represent $50 \mathrm{~nm}$. b, d EEL spectra of the bright dipolar mode of the devices in $\mathbf{a}$ and $\mathbf{c}$, demonstrating Rabi splitting. Green curves are experimental data, and orange curves are fits to the coupled oscillator model, as described in the Methods section. Insets demonstrate the points of excitation by the electron beam. $\mathbf{f B E M}$ simulation of the spectrum of the bright mode in a PC coupled to four QDs, as shown in $\mathbf{e}$.

criteria often discussed in the literature ${ }^{33}$. The first criterion, $g>$ $\left(\gamma_{\mathrm{p}}-\gamma_{\mathrm{e}}\right) / 4$ (where $g, \gamma_{\mathrm{p}}$ and $\gamma_{\mathrm{e}}$ are the coupling strength, plasmon linewidth and exciton linewidth, respectively), guarantees two real solutions in the coupled-oscillator model when the QD is resonantly tuned to the plasmon and may be seen as defining a lower bound for the strong-coupling regime. Put in a different way from the quantum mechanical point of view, when this criterion is fulfilled, the system has passed an exceptional point ${ }^{34,35}$ and is guaranteed to possess two distinct eigenstates. Based on the values of $\gamma_{\mathrm{p}}$ and $\gamma_{\mathrm{e}}$ we measure in our systems, the above criterion gives a value of $\sim 55 \mathrm{meV}$, and the values of $g$ extracted from our spectra (up to $85 \mathrm{meV}$ ) are larger.

While this criterion guarantees two eigenstates, it does not ensure that measured spectra will clearly show these states as separate peaks. Therefore, a second and stricter criterion is introduced. This criterion ${ }^{33}$, given by $g>\left(\gamma_{\mathrm{p}}+\gamma_{\mathrm{e}}\right) / 4$, is more heuristic, and requires a larger $g$ to be fulfilled. Indeed, in our case it gives a value of $\sim 120 \mathrm{meV}$, larger than the values we report
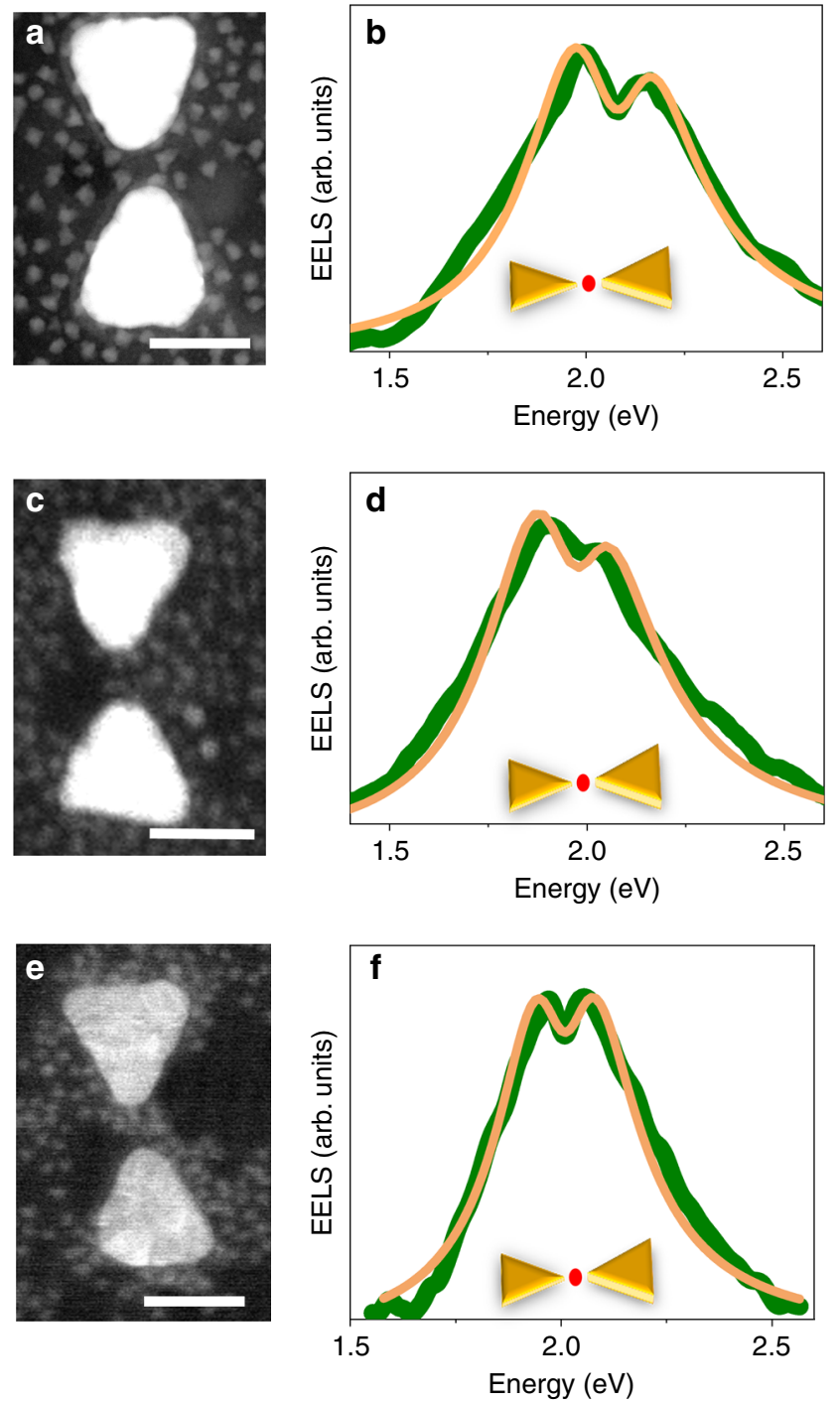

Fig. 3 Coupling with the dark dipolar mode. a, c, e ADF-STEM images of three devices loaded with QDs with an exciton energy of $2.0 \mathrm{eV}$. The scale bars represent $50 \mathrm{~nm}$. b, d, $\mathbf{f}$ EEL spectra of the dark mode of the devices in a, c and e demonstrating Rabi splitting, as with the bright mode. Green curves are experimental data, and orange curves are fits to the coupled oscillator model, as described in the Methods section. Insets demonstrate the points of excitation by the electron beam.

here, although not by a lot. However, in reality, the splitting of the two peaks in the spectrum grows continuously, as demonstrated by simulated spectra in Supplementary Fig. 4. The spectra are clearly split already when $g=55 \mathrm{meV}$ (i.e. the value of the first criterion), and the splitting increases as $g$ grows. Indeed, splitting is readily observed in the experimental spectra (Figs. 2 and 3). Therefore, we believe we can safely state that all the PC-QD systems measured here are found at the onset of the strongcoupling regime.

As noted already in the introductory remarks of this paper, the appeal of using dark (excitonic or plasmonic) modes rather than bright modes for quantum optical applications resides with their longer lifetimes. For example, a dark exciton of a self-assembled QD has been used as a qubit that emits clusters of entangled photons ${ }^{36}$. The lifetime of a dark plasmonic mode should be increased due to the lack of radiative damping, and this longer lifetime should affect the dynamics of coupling between a PC and quantum emitters ${ }^{31}$. Somewhat surprisingly, the longer lifetime 

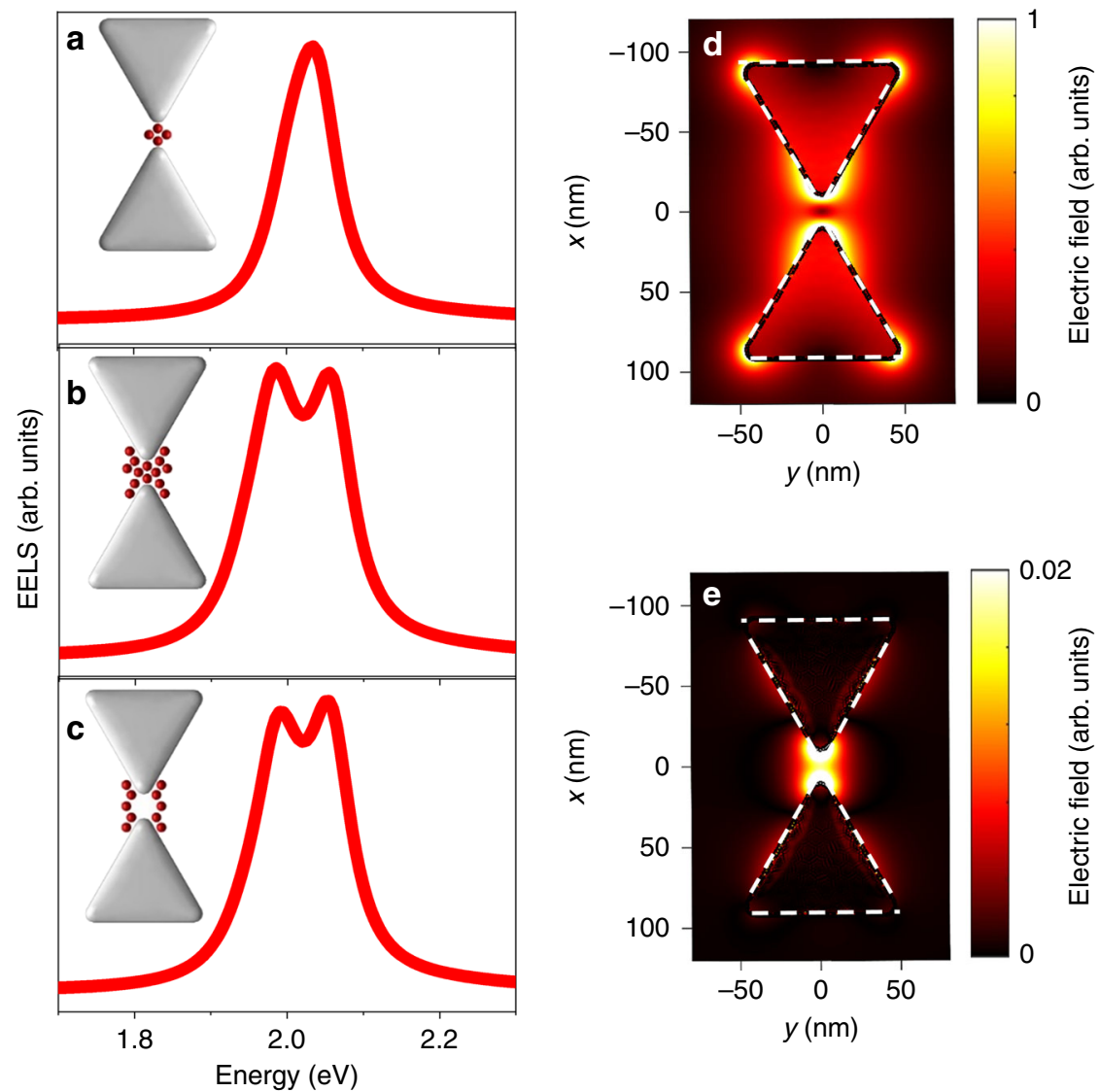

Fig. 4 EM simulations of dark mode coupling with the QDs. a Dark mode spectrum when four QDs are positioned in the center of the gap. $\mathbf{b}$ Dark mode spectrum when ten additional dots are added at the periphery of the gap. $\mathbf{c}$ Dark mode spectrum with QDs positioned only at the gap periphery. $\mathbf{d}$ Map of total electric field distribution around the PC at $2 \mathrm{eV}$, projected on the $x-y$ plane. e Map of the out-of-plane electric field distribution around the PC at $2 \mathrm{eV}$, projected on the $x-y$ plane. The maps in $\mathbf{d}$ and $\mathbf{e}$ were calculated for bowties without QDs. Note their different color scales.

does not lead to a significantly smaller linewidth of the dark mode peak in our EEL spectra (compared to the bright mode, Fig. 1). This might point to factors that dephase the excitation of the dark mode and therefore contribute to its linewidth significantly without affecting its lifetime. This possibility likely requires timedependent studies with femtosecond resolution to be tested. Future experimental work might be able to probe the relation between the long lifetime of the dark mode and Rabi oscillations in the coupled PC-QD system. If the involvement of emissive QDs makes the dark plasmon-exciton polaritons partially emissive, this phenomenology might be detected in the far field using time-resolved cathodoluminescence ${ }^{37}$.

\section{Methods}

Materials. Electron microscopy grids with 18-nm-thick $\mathrm{SiO}_{2}$ suspended films were purchased from Ted Pella Inc. A poly(methyl methacrylate) 950K A2 (PMMA) electron beam resist, used for the fabrication of bowties, was obtained from MicroChem, as was the solvent stripper Remover PG. Chromium (used as an adhesive layer) and silver were obtained from Kurt J. Lesker. Water-soluble mercapto-undecanoic acid capped CdSe/ZnS core/shell nanocrystals (QDs) were acquired from MK Impex Corp. Thrimethylaluminum (TMA), used as a precursor for atomic layer deposition, was purchased from Strem Chemicals Inc.

Electron beam lithography and fabrication of silver bowties. PMMA was spincoated on the grids at a speed of $5000 \mathrm{rpm}$ for $50 \mathrm{~s}$ to achieve a thickness of $60 \mathrm{~nm}$, followed by baking at $180^{\circ} \mathrm{C}$ for $90 \mathrm{~s}$. The PMMA-coated grids were loaded into a Raith E_line Plus electron-beam lithography system and the PMMA was exposed to define the shape of bowties. The accelerating voltage used for the exposure was $30 \mathrm{kV}$ and the beam current was $30 \mathrm{pA}$. The design consisted of matrices of bowties, with each matrix hosting 12 bowties. Each bowtie was separated by $10 \mu \mathrm{m}$ from its neighbors. To remove the exposed PMMA, substrates were developed using 1:3 methyl isobutyl ketone:isopropyl alcohol for $30 \mathrm{~s}$, followed by immersion in a stopper (isopropyl alcohol) for $30 \mathrm{~s}$ and drying with a nitrogen flow. An electron-beam evaporator (Odem) was used for metal deposition on the patterned substrates. Initially, chromium was evaporated to deposit a 2-nm adhesion layer, followed by silver. The lengths of the silver prisms generated in this way ranged from 65 to $85 \mathrm{~nm}$, and their thickness was $25 \mathrm{~nm}$. After metallization, a lift-off step was performed in a solvent stripper for $40 \mathrm{~min}$ to obtain silver bowties on the $\mathrm{SiO}_{2}$ films. To protect the bowties from oxidation, we deposited on the grid a conformal thin layer of alumina $(2.5 \mathrm{~nm})$ using an atomic layer deposition system (Fiji Plasma), with TMA as a precursor. Finally, PMMA was again spin coated on the grid in order to protect the bowtie structure from degradation during the incorporation of QDs, as the alumina layer was found not to be sufficient in protecting the bowties within a water-based solution.

Incorporation of QDs into bowtie structures. We first made the PMMA surface hydrophilic by applying a delicate $\mathrm{N}_{2}$ plasma using an inductively coupled plasma apparatus (Plasma Therm Ltd.). We then immersed the sample vertically in a water-based solution of QDs and allowed the solution to evaporate within a low vacuum environment. During the evaporation, QDs were randomly positioned on the PMMA surface. In the last stage, we immersed the grid in acetone to dissolve the resist. Under the conditions we used (unheated remover and a thin resist), the QDs were not completely washed away with the resist, and the majority of the QDs tended to randomly deposit on the substrate, sticking in the vicinity of the bowties.

EEL measurements. Electron microscopy data were recorded using a double aberration-corrected Themis Z microscope (Thermo Fisher Scientific Electron Microscopy Solutions, Hillsboro, USA) equipped with a high-brightness Schottky field emission gun and a Wien-type monochromator at an accelerating voltage of $80 \mathrm{kV}$. EDS hyperspectral data were obtained with a Super-X G2 four-segment SDD detector with a probe semi-convergence angle of $30 \mathrm{mrad}$ and beam current of $\sim 200 \mathrm{pA}$. Low-loss EEL maps were recorded on a Gatan Quantum GIF 966ERS energy loss spectrometer (Gatan Inc., Pleasanton, USA) with an Ultrascan1000 
CCD camera. The low-loss EEL maps were recorded using a monochromated STEM probe with an energy width of $70-80 \mathrm{meV}$, a semi-convergence angle of 34 mrad and a beam current of $160 \mathrm{pA}$. The outer semi-collection angle of the spectrometer was set to $18 \mathrm{mrad}$.

Data analysis was performed in the software DigitalMicrograph (Gatan Inc., Pleasanton, USA). In all figures, the zero-loss peak was removed by subtracting its part to the left of the $y$ axis, reflected to the right side of the axis, from the whole spectrum. In Figs. 2 and 3, the spectra of specific plasmonic modes were isolated by first subtracting a background signal measured far from the plasmonic structures and then substracting the contribution of neighboring modes after fitting them to Gaussian curves. Raw spectra were smoothed using a Savitzky-Golay filter.

EM simulations. Numerical simulations of EEL maps and spectra were performed using the boundary-element method as implemented in the Matlab toolbox MNPBEM $^{26}$. A numerical model of a bowtie was constructed from two equilateral triangular prisms with an edge length of 73 or $87 \mathrm{~nm}$, a height of $20 \mathrm{~nm}$, radii of curvature at the vertices of $7 \mathrm{~nm}$, and a gap size of $20 \mathrm{~nm}$. The edge lengths were selected to match the experimental resonance frequency for the bright and dark modes. The complex refractive index of silver from Johnson and Christy ${ }^{38}$ was used, and a refractive index of 1.35 was assumed for the ambient medium. The bowtie was excited by an electron beam (energy $80 \mathrm{keV}$ ) at normal incidence. Simulations of EEL spectra for a bowtie loaded with QDs were performed with dots modeled as spheres of $8 \mathrm{~nm}$ located within the gap and its periphery. The complex dielectric function of these dots was approximated by a Lorentz model (similarly to our previous work ${ }^{11}$ ) with a high-frequency dielectric constant $\epsilon_{\infty}=6.0$, $\omega_{0, \text { bright }}=1.75 \mathrm{eV}, \omega_{0, \text { dark }}=1.99 \mathrm{eV}, \gamma_{0}=0.08 \mathrm{eV}$, and an oscillator strength $f=$ 0.8 .

Spectral fitting with the coupled oscillator model. EEL spectra were fitted with the expression for the extinction of two coupled oscillators at frequency $\omega^{25}$ :

$$
S(\omega) \propto \omega \operatorname{Im}\left(\frac{\left(\omega_{\mathrm{e}}^{2}-\omega^{2}-i \sqrt{\left(\gamma_{\mathrm{e}}^{2}+\delta^{2}\right)} \omega\right)}{\left(\omega^{2}-\omega_{\mathrm{p}}^{2}+i \sqrt{\left(\gamma_{\mathrm{p}}^{2}+\delta^{2}\right)} \omega\right)\left(\omega^{2}-\omega_{\mathrm{e}}^{2}+i \sqrt{\left(\gamma_{\mathrm{e}}^{2}+\delta^{2}\right)} \omega\right)-4 \omega^{2} g^{2}}\right)
$$

where $\omega_{\mathrm{e}}, \omega_{\mathrm{p}}, \gamma_{\mathrm{e}}$ and $\gamma_{\mathrm{p}}$ are the resonance frequencies and linewidths of the emitter and cavity, respectively, and $g$ is the coupling constant. We fixed the values of $\gamma_{\mathrm{e}}$ $(110-130 \mathrm{meV})$ and $\gamma_{\mathrm{p}}(350-395 \mathrm{meV})$, based on the linewidths of the photoluminescence spectra of individual QDs and the scattering spectra of empty bowties (it was found that these linewidths slightly varied between devices). We broadened the linewidths of the emitter and the cavity by the instrumental broadening $\delta(70 \mathrm{meV})$.

\section{Data availability}

All relevant data are available from the authors upon request.

Received: 29 October 2019; Accepted: 2 January 2020;

Published online: 24 January 2020

\section{References}

1. Haroche, S. \& Raimond, J. M. Exploring the Quantum: Atoms, Cavities and Photons. 1st edn (Oxford University Press, 2006).

2. Hennessy, K. et al. Quantum nature of a strongly coupled single quantum dotcavity system. Nature 445, 896-899 (2007).

3. Monroe, C. Quantum information processing with atoms and photons. Nature 416, 238-246 (2002).

4. Lo, H. K. \& Chau, H. F. Unconditional security of quantum key distribution over arbitrarily long distances. Science 283, 2050-2056 (1999).

5. Kimble, H. J. The quantum internet. Nature 453, 1023-1030 (2008).

6. Zhong, X. L. et al. Energy transfer between spatially separated entangled molecules. Angew. Chem. Int. Ed. 56, 9034-9038 (2017).

7. Thomas, A. et al. Tilting a ground-state reactivity landscape by vibrational strong coupling. Science 363, 616-+ (2019).

8. Halas, N. J., Lal, S., Chang, W. S., Link, S. \& Nordlander, P. Plasmons in strongly coupled metallic nanostructures. Chem. Rev. 111, 3913-3961 (2011).

9. Haran, G. \& Chuntonov, L. Artificial plasmonic molecules and their interaction with real molecules. Chem. Rev. 118, 5539-5580 (2018).

10. Hugall, J. T., Singh, A. \& van Hulst, N. F. Plasmonic cavity coupling. ACS Photonics 5, 43-53 (2018).

11. Santhosh, K., Bitton, O., Chuntonov, L. \& Haran, G. Vacuum Rabi splitting in a plasmonic cavity at the single quantum emitter limit. Nat. Commun. 7, ncomms11823 (2016).

12. Groß, H., Hamm, J. M., Tufarelli, T., Hess, O. \& Hecht, B. Near-field strong coupling of single quantum dots. Sci. Adv. 4, eaar4906 (2018).
13. Leng, H., Szychowski, B., Daniel, M. C. \& Pelton, M. Strong coupling and induced transparency at room temperature with single quantum dots and gap plasmons. Nat. Commun. 9, 4012 (2018).

14. Herzog, J. B. et al. Dark plasmons in hot spot generation and polarization in interelectrode nanoscale junctions. Nano Lett. 13, 1359-1364 (2013).

15. Gomez, D. E. et al. The dark side of plasmonics. Nano Lett. 13, 3722-3728 (2013).

16. Garcia de Abajo, F. J. Optical excitations in electron microscopy. Rev. Mod. Phys. 82, 209-275 (2010).

17. Koh, A. L. et al. Electron energy-loss spectroscopy (EELS) of surface plasmons in single silver nanoparticles and dimers: influence of beam damage and mapping of dark modes. ACS Nano 3, 3015-3022 (2009).

18. Koh, A. L., Fernandez-Dominguez, A. I., McComb, D. W., Maier, S. A. \& Yang, J. K. W. High-resolution mapping of electron-beam-excited plasmon modes in lithographically defined gold nanostructures. Nano Lett. 11, 1323-1330 (2011)

19. Cherqui, C., Thakkar, N., Li, G. L., Camden, J. P. \& Masiello, D. J. Characterizing localized surface plasmons using electron energy-loss spectroscopy. Annu. Rev. Phys. Chem. 67, 331-357 (2016).

20. Colliex, C., Kociak, M. \& Stephan, O. Electron Energy Loss Spectroscopy imaging of surface plasmons at the nanometer scale. Ultramicroscopy $\mathbf{1 6 2}$, A1-A24 (2016).

21. Losquin, A. et al. Unveiling nanometer scale extinction and scattering phenomena through combined electron energy loss spectroscopy and cathodoluminescence measurements. Nano Lett. 15, 1229-1237 (2015).

22. Bernasconi, G. D. et al. Where does energy go in electron energy loss spectroscopy of nanostructures? ACS Photonics 4, 156-164 (2017)

23. Liu, M. Z., Lee, T. W., Gray, S. K., Guyot-Sionnest, P. \& Pelton, M. Excitation of dark plasmons in metal nanoparticles by a localized emitter. Phys. Rev. Lett. 102, 107401 (2009).

24. Efros, A. L. \& Rosen, M. The electronic structure of semiconductor nanocrystals. Annu. Rev. Mater. Sci. 30, 475-521 (2000).

25. Wu, X., Gray, S. K. \& Pelton, M. Quantum-dot-induced transparency in a nanoscale plasmonic resonator. Opt. Express 18, 23633-23645 (2010).

26. Hohenester, U. \& Trugler, A. MNPBEM-A Matlab toolbox for the simulation of plasmonic nanoparticles. Comput. Phys. Commun. 183, 370-381 (2012).

27. Hohenester, U. Simulating electron energy loss spectroscopy with the MNPBEM toolbox. Comput. Phys. Commun. 185, 1177-1187 (2014).

28. Wei, J. K., Jiang, N., Xu, J., Bai, X. D. \& Liu, J. Y. Strong coupling between $\mathrm{ZnO}$ excitons and localized surface plasmons of silver nanoparticles studied by STEM-EELS. Nano Lett. 15, 5926-5931 (2015).

29. Yankovich, A. B. et al. Visualizing spatial variations of plasmon-exciton polaritons at the nanoscale using electron microscopy. Nano Lett. 19, 8171-8181 (2019)

30. Li, R. Q., Garcia-Vidal, F. J. \& Fernandez-Dominguez, A. I. Plasmon-exciton coupling in symmetry-broken nanocavities. ACS Photonics 5, 177-185 (2018).

31. Demetriadou, A. et al. Spatiotemporal dynamics and control of strong coupling in plasmonic nanocavities. ACS Photonics 4, 2410-2418 (2017)

32. Ramezani, M. et al. Plasmon-exciton-polariton lasing. Optica 4, 31-37 (2017)

33. Torma, P. \& Barnes, W. L. Strong coupling between surface plasmon polaritons and emitters: a review. Rep. Prog. Phys. 78, 013901 (2015).

34. Miri, M. A. \& Alu, A. Exceptional points in optics and photonics. Science 363 42 (2019).

35. Rodriguez, S. R. K. Classical and quantum distinctions between weak and strong coupling. Eur. J. Phys. 37, 025802 (2016).

36. Schwartz, I. et al. Deterministic generation of a cluster state of entangled photons. Science 354, 434-437 (2016).

37. Meuret, S. et al. Complementary cathodoluminescence lifetime imaging configurations in a scanning electron microscope. Ultramicroscopy 197, 28-38 (2019).

38. Johnson, P. B. \& Christy, R. W. Optical constants of the noble metals. Phys Rev. B 6, 4370-4379 (1972).

\section{Acknowledgements}

G.H. is the incumbent of the Hilda Pomeraniec Memorial Professorial Chair. This work was partially supported by EU project 810626 - SINNCE.

\section{Author contributions}

O.B., S.N.G. and G.H. designed the research. O.B., S.N.G. and L.H. performed the experiments. M.K., V.K. and T.S. performed EM simulations. All authors participated in writing the manuscript.

\section{Competing interests}

The authors declare no competing interests. 


\section{Additional information}

Supplementary information is available for this paper at https://doi.org/10.1038/s41467020-14364-3.

Correspondence and requests for materials should be addressed to G.H.

Peer review information Nature Communications thanks Matthew Pelton and the other, anonymous, reviewer(s) for their contribution to the peer review of this work.

Reprints and permission information is available at http://www.nature.com/reprints

Publisher's note Springer Nature remains neutral with regard to jurisdictional claims in published maps and institutional affiliations. (c) Open Access This article is licensed under a Creative Commons Attribution 4.0 International License, which permits use, sharing, adaptation, distribution and reproduction in any medium or format, as long as you give appropriate credit to the original author(s) and the source, provide a link to the Creative Commons license, and indicate if changes were made. The images or other third party material in this article are included in the article's Creative Commons license, unless indicated otherwise in a credit line to the material. If material is not included in the article's Creative Commons license and your intended use is not permitted by statutory regulation or exceeds the permitted use, you will need to obtain permission directly from the copyright holder. To view a copy of this license, visit http://creativecommons.org/licenses/by/4.0/.

(C) The Author(s) 2020 